\title{
Diet induced weight loss reduces mean platelet volume in people with obesity
}

\author{
${\text { Huseyin } \text { Kurt }^{1 *} \text {, Davut Demirkiran }}^{1}$
}

\begin{abstract}
Objectives: To investigate is there a relationship between obesity parameters, mean platelet volume (MPV) and neutrophil lymphocyte ratio (NLR), and to examine is there a change in MPV and NLR level with weight loss.

Material and Methods: The retrospective study consisted of two groups as the group losing weight after 24 weeks of diet (WLG) and the control group not losing weight (CG). Body mass index (BMI), MPV, NLR and lipid values of each group were recorded at the beginning and at the end of the study.

Results: The study consisted of 186 individuals 94 of whom were in WLG and 92 were in CG. There was a statistically significant positive correlation between the initial value of MPV and BMI in WLG $(\mathrm{p}<0.05)$. There was a significant difference existed in the initial and final lipid parameters of WLG $(\mathrm{p}<0.0001)$. Changes in initial and final MPV values in WLG and CG were different from each other $(\mathrm{p}<0.01)$. The difference between the initial and final MPV values and BMI values in WLG was statistically significant $(\mathrm{P}<0.001)$. No significant correlation was found between obesity and weight loss with NLR.
\end{abstract}

Conclusions: There was a statistically significant correlation between MPV and BMI. MPV levels decreased in obese individuals with weight loss. No correlation existed between BMI and NLR.

Keywords: Diet, weight loss, mean platelet volume, neutrophil lymphocyte ratio, obesity, body mass index

\section{Introduction}

The importance of inflammatory markers is increasing as coronary artery diseases are currently the most common causes of death in the world, which is related to inflammation (1). Therefore, a number of studies have recently been conducted on inflammation and biomarkers which reflect inflammatory conditions (1, 2). In obesity whose prevalence is gradually increasing due to the decrease in physical activity and increase in high-energy food consumption, body mass index (BMI) above normal increases mortality and morbidity (3, 4). According to World Health Organization (WHO), an individual is defined as overweight (preobese) if BMI is above $25 \mathrm{~kg} / \mathrm{m}^{2}$ and as obese if BMI is above $30 \mathrm{~kg} / \mathrm{m}^{2}$ (4). Furthermore, obesity plays an important role in the formation of many pathological conditions. The most frequent conditions can be listed as cardiovascular disease, cerebrovascular disease, hypertension (HT), diabetes mellitus (DM), dyslipidemia, infertility, certain types of cancer such as prostate, breast and colon (5).

Although obesity-related causes of inflammation are not fully understood, it is estimated that it occurs due to direct activation of immune cells in the circulatory system $(6,7)$.
The inflammatory process also occurs in liver, pancreas, adipose and muscle tissue with circulation. Adipose tissue has both initiatory and contributory role in systemic inflammation $(6,7)$.

Mean platelet volume (MPV) associated with cardiovascular and cerebrovascular diseases and accepted as an indication of atherosclerosis is a parameter used to evaluate the size and activity of platelets (8). The volume of activated platelets increases, which causes MPV rise. Large platelets including more granules and produces more vasoactive and prothrombotic factors make more aggregation compared to small platelets $(9,10)$. MPV is known to increase in acute myocardial infarction, acute ischemic stroke, preeclampsia and renal artery stenosis (8). Additionally, in some studies, it has been discovered that MPV is positively correlated with

BMI and high in obesity (11). In recent studies on humans and animals, it has been demonstrated that the change in neutrophil and lymphocyte numbers poses a potential risk for the development of obesity-related metabolic disorders $(12,13)$.

Received 11-01-2015, Accepted 22-01-2015, Available Online 15-02-2016

1 Department of Internal Medicine, School of Medicine, The University of Balikesir, Turkey

*Corresponding Author: Huseyin Kurt E-mail: hsynkurt@yahoo.com 
It is believed that Neutrophil / Lymphocyte ratio (NLR) is a marker of systemic inflammation and has a prognostic significance for cardiovascular diseases. These hematological markers are important in terms of making assessment without creating extra costs during the complete blood count (14). Although there are a number of studies conducted on the relationship between MPV and obesity and no common conclusion is drawn. In this study, our aim is to investigate the relationship between obesity parameters, which are known to accelerate the development of atherothrombosis and the incidence of cardiovascular mortality and morbidity, MPV and NLR. We also aim to examine whether there is a change in MPV and NLR levels with weight loss thanks to a diet plan in obese individuals.

\section{Material and Methods}

This study was conducted retrospectively after the approval of Ethics Committee of Balıkesir University School of Medicine was granted. When individuals go to Balıkesir University School of Medicine Health Application and Research Hospital with complaints related to being overweight, the arterial blood pressure is measured and physical examination is performed. After those procedures, complete blood count and biochemical tests (12 hour fasting glucose, total cholesterol, LDL cholesterol, HDL cholesterol, triglycerides, and HOMA-IR TSH) are performed routinely. For complete blood count, blood sample is taken from the antecubital vein, put into tubes with EDTA and studied with Beckman Coulter LH 780 Hematology Analyzer (Beckman Coulter, Inc., CA, USA) device within an hour. If metabolic syndrome (MS), DM, and dyslipidemia are detected in those individuals medical treatment is given and they are directed to diet clinic. Measuring weight and height, BMI of obese individuals are calculated through weight $(\mathrm{kg}) /$ (height $)^{2}\left(\mathrm{~m}^{2}\right)$ formulas, and appropriate nutrition programs are given by dieticians. Weight changes of these individuals are followed for 12-week periods. During the controls in internal medicine clinic, detailed physical examination, complete blood count and biochemical tests are performed, and individuals meet the dieticians again. We scanned the files of obese individuals who were directed to dieticians between 2013-2015, had $\geqslant 30 \mathrm{~kg} / \mathrm{m} 2$ body mass index (BMI) according to WHO classification, and followed at least 24 weeks of dieting. The individuals who were found to have DM (fasting blood glucose $\geqslant 126$ and hemoglobin A1c> $6.4 \%$ ), cardiovascular disease, HT (systolic blood pressure $\geqslant 140 \mathrm{~mm} \mathrm{Hg}$ diastolic blood pressure $\geqslant 90$ ), cerebrovascular disease, liver failure, renal failure, smoking and alcohol habit were excluded from the study. On the other hand, individuals who were eligible for the study were divided into two groups as weight loss group (WLG) including individuals with body weight change higher than $5 \%$, and the control group (CG) consisting of individuals who had weight loss less than $5 \%$. The initial values of WLG and CG were recorded as MPV1, NLR1, BMI1 and the values after 24 weeks were recorded as MPV2, NLR2 and BMI2.

\section{Diet Plan}

Patients' daily energy requirements were calculated according to age, gender, weight and height. A weight loss diet was implemented ensuring that energy intake was above the basal metabolic rate (BMR). Gerrior et al. formulae was used to calculate BMR: "BMR $=247$ $-2.67 \times$ age $($ year $)+401,5 \times$ height boy (meter) $+8,6$ $\times$ weight $(\mathrm{kg})$ " (15). The diet consisted of $55-60 \%$ carbohydrates $12-15 \%$ protein and $25-30 \%$ lipid and the energy value of the diets given ranged between1300-1800 kcal.

Statistical analysis: The values of the obese group losing weight as a result of dieting and control group losing weight without diet were evaluated using SPSS software version 20.0 (SPSS Inc, Chicago, IL, USA). Chi square test was used for determining the relations of gender and age between groups. In addition, groups were compared using the Student's t-test. The groups' initial and final results were compared using the Paired sample $t$ test. The correlation between BMI, MPV and NLR was calculated via Pearson correlation test. MPV changes within the group were demonstrated through univariate covariance analysis. $\mathrm{P}<0.05$ value was accepted as significant.

\section{Results}

186 obese individuals (153 females, 33 males) appropriate for the study criteria were included in the study. WLG consisted of 94 individuals (79 females and 15 males) between 21-64 years old, while CG included 92 individuals ( 74 females and 18 males) between 19-64 years old. No difference was found between groups in terms of gender and age distribution $(\mathrm{P}>0.05)$. Moreover, no significant difference existed between MPV values of males and females $(\mathrm{P}>0.05)$. The mean MPV1 level of all individuals in the study was found to be $8.38 \pm 0.95$ while the mean MPV2 was $8.21 \pm 0.94$. The mean MPV1 of WLG was $8.40 \pm 0.95$ whereas the mean MPV2 was found to be $7.93 \pm 0.84$ (Table 1). When the initial BMI values of both groups were compared, a significant difference was found $(p<0.05)$. The mean BMI 1 value of WLG was $36.68 \pm 5.72$, whereas it was $34.62 \pm 5.09$ for CG. At the end of the study, when the mean BMI 2 of WLG $(32.17 \pm 5.04)$ and the 
Table 1: The anthropometric measurements and haematological values of non-weight loss and weight loss groups

\begin{tabular}{lcccccc} 
& \multicolumn{2}{c}{ Non weight loss (n: 97) } & \multicolumn{3}{c}{ Weight loss (n: 101) } \\
& First count & Last count & p & First count & Last count & p \\
\hline Body weight (kg) & $89.0 \pm 14.2$ & $90.5 \pm 14.5$ & 0.001 & $94.3 \pm 15.3$ & $82.8 \pm 14.0$ & 0.001 \\
BMI (kg/m2) & $34.6 \pm 5.0$ & $35.2 \pm 5.1$ & 0.001 & $36.6 \pm 5.7$ & $32.1 \pm 5.0$ & 0.001 \\
Total cholesterol (mg/dL) & $224.1 \pm 46.7$ & $217.7 \pm 43.2$ & 0.137 & $239.1 \pm 55.0$ & $190.1 \pm 39.8$ & 0.001 \\
LDL- cholesterol (mg/dL) & $138.9 \pm 42.2$ & $139.7 \pm 36.5$ & 0.161 & $152.5 \pm 52.5$ & $107.4 \pm 33.9$ & 0.001 \\
HDL- cholesterol (mg/dL) & $50.2 \pm 12.1$ & $49.6 \pm 11.2$ & 0.462 & $50.8 \pm 11.6$ & $56.4 \pm 10.4$ & 0.001 \\
Triglyceride (mg/dL) & $175.1 \pm 65.6$ & $170.7 \pm 65.6$ & 0.359 & $190.3 \pm 85.8$ & $134.0 \pm 55.1$ & 0.001 \\
MPV (fl) & $8.3 \pm 0.9$ & $8.5 \pm 0.9$ & 0.023 & $8.4 \pm 0.9$ & $7.9 \pm 0.8$ & 0.001 \\
NLR (\%) & $1.6 \pm 0.9$ & $1.7 \pm 1.2$ & 0.405 & $1.8 \pm 0.9$ & $1.9 \pm 0.8$ & 0.001 \\
\hline
\end{tabular}

BMI: body mass index; LDL: Low Density Lipoprotein; HDL: High Density Lipoprotein;

MPV: mean platelet volume; NLR: neutrophil lymphocyte ratio

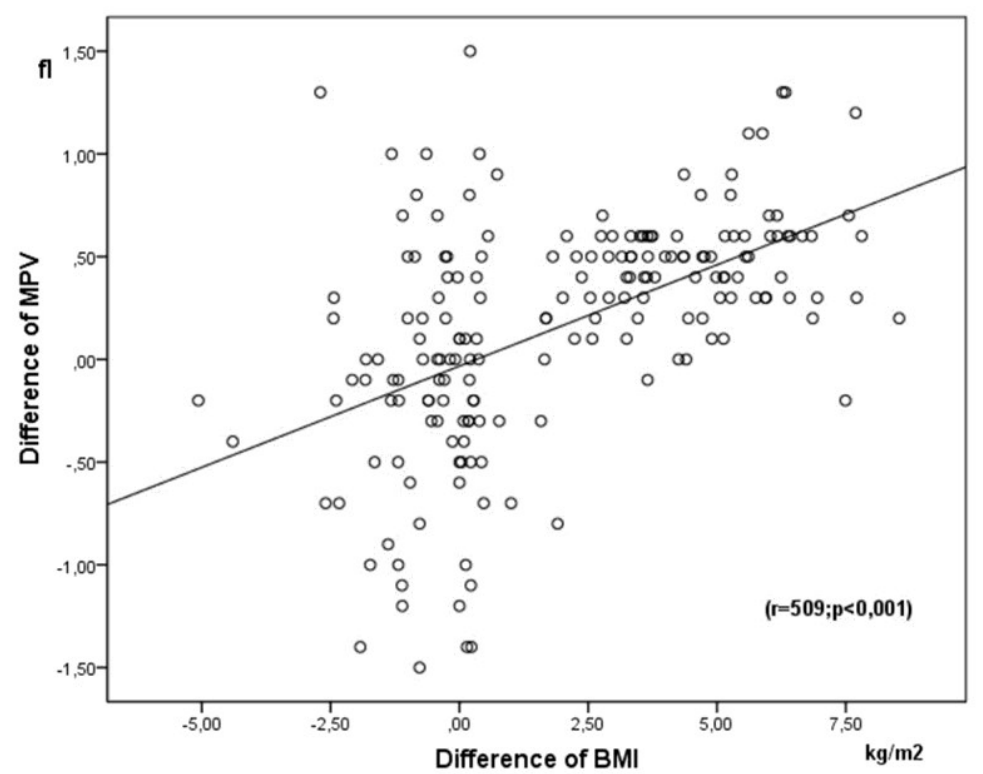

Figure 1: The correlation between the initial and final body mass index and mean platelet volume values

mean BMI2 of CG $(35.20 \pm 5.19)$ were compared, no significant difference was found $(\mathrm{p}>0.05)$.

While no difference existed in the initial and final lipid parameters of $\mathrm{CG}(\mathrm{p}>0.05)$, a significant difference existed in the initial and final lipid parameters of WLG $(p<0.0001)$ (Table 1). A positive correlation existed between MPV and BMI values of all individuals in the study $(\mathrm{r}=0.140 ; \mathrm{p}=0.05)$. When this correlation was examined separately for both groups, it was found that there was a significant difference between BMI1 and MPV1 in WLG ( $\mathrm{r}$ $=0.267 ; \mathrm{p}<0.01)$, whereas no difference was found in CG.

While no difference existed in the initial mean MPV values of each group, a significant difference was found at the end of the study $(\mathrm{p}<0.001)$. The reason of this result was to be decrease of MPV in WLG at the end.

Medical Science and Discovery 2016; 3(2): 98-102
It was discovered through univariate covariance analysis test that the change in initial and final measurement of MPV values of each individual in WLG was different from the change in CG $(p<0.01)$.

There was a statistically significant correlation between the initial and final MPV and BMI values of individuals in the study $(r=0.509 ; p<0.001)$ (Figure 1). No correlation was found between NLR and BMI for both WLG and CG. Additionally, there was no correlation between weight loss and NLR ( $p>0.05)$.

\section{Discussion}

In previous studies, it was stated that the level of obesity and MS are associated with inflammation and thus cause atherosclerosis (16). MPV which indicates platelet activity is associated with the pathological conditions in thrombopoiesis $(16,17)$. MPV levels in obese individuals are higher than non-obese individuals. 
Coban et al. demonstrated in their study that MPV is higher in obese individuals than non-obese individuals (11). The results of studies examining the relationship of MPV with obesity and MS are incompatible with each other.

In a comprehensive study conducted by Kutlucan et al., it was found that MPV values of obese individuals with MS were different from values of obese individuals without MS (18). They claimed that MPV levels are more affected by obesity than MS. On the other hand, Tavil et al. stated that MPV is higher in MS and the reason for this was that it is related to HT, waist circumference, BMI, and fasting plasma glucose (19).

In literature, there are many studies in parallel with our study investigating the impact of diet on MPV in obese individuals. In the study conducted by Toplak et al., weight loss with hypocaloric diet and low calorie diet was examined. It was found in that study that MPV values increased in the first 8 weeks compared to the initial value, and MPV levels of individuals losing weight after 48 weeks dropped to the initial level. MPV did not change compared to initial level and no change was observed in lipid values (20). In our study, it was found that MPV and lipid values decreased after weight loss. When we compare our study and the study conducted by Toplak et al., it can be said that lipid levels have an important role in the effect of weight loss on MPV levels. In a study conducted by Coban et al., obese and non-obese individuals were compared. They found that MPV values in obese individuals were higher than nonobese individuals, and MPV values measured after weight loss whit diet decreased compared to the initial MPV values (21). However, the non-existence of control group consisted of obese individual without weight loss is the major limitation of that study. Unlike those studies, we investigated the changes in MPV levels of two groups including individuals with weight loss and without weight loss following the same diet. It was discovered that MPV levels decreased in obese individuals even if obesity continued after at least $\% 5$ weight losses.

Positive correlation existed between BMI and MPV values of individuals who participated in the study at the beginning. When this correlation is examined separately for both groups, it was discovered there was a significant correlation between BMI and MPV in WLG. However, no correlation existed between BMI and MPV in CG. Coban et al. stated that there was a significant correlation between BMI and MPV levels of obese individuals who lost weight, which is similar to our results (21). In addition, we found a positive correlation between the initial and final weight changes of individuals and the initial and final BMI changes of individuals (Figure 1). Accordingly, it can be said that MPV values decrease when individuals lose weight. While the mean MPV value of both groups was similar, the mean BMI value of WLG was higher than CG. At the end of the study, the mean BMI value of both groups was similar due to weight loss in WLG. Yet, the mean MPV value of WLG was lower than the mean MPV value of CG. Even if the mean BMI was similar, the likely reason of declining in MPV could be the decrease in lipid level of WLG. These findings suggest that MPV, which is an atherosclerotic marker in obese individuals, are more affected from lipid changes than weight loss.

There is no consensus about the use of NLR as a marker similar to MPV. NLR is an indicator of systemic inflammation, and there exists several studies asserting that it provides survey estimation after coronary intervention (22) and coronary artery bypass grafting operations (14). In the study conducted by Ryder et al., they found that NLR was not a useful marker in obese individuals (23). When Agacayak et al examined NLR in obese individuals with polycystic ovary syndrome (PCO), and obese individuals without PCO, they discovered that NLR was higher in obese individuals with PCO and they stated that high NLR might be a contributor in the formation of cardiovascular disease in individuals with PCO (24).

Bahadir et al claimed that NLR was lower in individuals who had a low BMI level than individuals who had a high BMI level. But it was not statistically significant (25). Additionally, in that study, it was reported that NLR, itself, could not be a reliable marker of inflammation, and did not differ according to the degree of obesity (25). Similar to that study, we found that there was no difference between the initial NLR value and the value after weight loss and NLR was not affected by BMI.

The present study had several limitations. First, design of the study is retrospective study. Obese individuals with no chronic diseases can investigate with prospective studies in the future. Second, our findings are based only on the Turkish population; different results might be observed in other ethnic groups.

\section{Conclusion}

As a conclusion even if obesity continues after at least $5 \%$ weight loss, MPV levels reduce. The changes in MPV values are profoundly affected by the changes of lipid values.

Acknowledgments: The authors thank to dietician Hayrettin Kara for his help in the process of collecting data for the study.

Conflict of Interest: The authors declare that they have no competing interest. 


\section{References}

1. Folsom AR, Aleksic N, Catellier D, Juneja HS, Wu KK. Creactive protein and incident coronary heart disease in the Atherosclerosis Risk In Communities (ARIC) study. American heart journal. 2002;144(2):233-8.

2. Folsom AR, Wu KK, Rosamond WD, Sharrett AR, Chambless LE. Prospective study of hemostatic factors and incidence of coronary heart disease: the Atherosclerosis Risk in Communities (ARIC) Study. Circulation. 1997;96(4):1102-8.

3. Adams KF, Schatzkin A, Harris TB, Kipnis V, Mouw T, Ballard-Barbash R, et al. Overweight, obesity, and mortality in a large prospective cohort of persons 50 to 71 years old. The New England journal of medicine. 2006;355(8):763-78.

4. Waxman A. Prevention of chronic diseases: WHO global strategy on diet, physical activity and health. Food and nutrition bulletin. 2003;24(3):281-4

5. Poirier P, Giles TD, Bray GA, Hong Y, Stern JS, Pi-Sunyer FX, et al. Obesity and cardiovascular disease: pathophysiology, evaluation, and effect of weight loss: an update of the 1997 American Heart Association Scientific Statement on Obesity and Heart Disease from the Obesity Committee of the Council on Nutrition, Physical Activity, and Metabolism. Circulation. 2006;113(6):898-918

6. Elgazar-Carmon V, Rudich A, Hadad N, Levy R. Neutrophils transiently infiltrate intra-abdominal fat early in the course of high-fat feeding. Journal of lipid research. 2008;49(9):1894-903.

7. Pecht T, Gutman-Tirosh A, Bashan N, Rudich A Peripheral blood leucocyte subclasses as potential biomarkers of adipose tissue inflammation and obesity subphenotypes in humans. Obesity reviews : an official journal of the International Association for the Study of Obesity. 2014;15(4):322-37

8. Bath PM, Butterworth RJ. Platelet size: measurement, physiology and vascular disease. Blood coagulation \& fibrinolysis : an international journal in haemostasis and thrombosis. 1996;7(2):157-61.

9. Cay S, Biyikoglu F, Cihan G, Korkmaz S. Mean platelet volume in the patients with cardiac syndrome X. Journal of thrombosis and thrombolysis. 2005;20(3):175-8.

10. Jagroop IA, Clatworthy I, Lewin J, Mikhailidis DP. Shape change in human platelets: measurement with a channelyzer and visualisation by electron microscopy. Platelets. 2000;11(1):28-32.

11. Coban E, Ozdogan M, Yazicioglu G, Akcit F. The mean platelet volume in patients with obesity. International journal of clinical practice. 2005;59(8):981-2

12. Talukdar S, Oh da Y, Bandyopadhyay G, Li D, Xu J, $\mathrm{McNelis} \mathrm{J}$, et al. Neutrophils mediate insulin resistance in mice fed a high-fat diet through secreted elastase. Nature medicine. 2012;18(9):1407-12

13. Dixon JB, O'Brien PE. Obesity and the white blood cell count: changes with sustained weight loss. Obesity surgery. 2006;16(3):251-7.
14. Gibson PH, Croal BL, Cuthbertson BH, Small GR, Ifezulike AI, Gibson G, et al. Preoperative neutrophillymphocyte ratio and outcome from coronary artery bypass grafting. American heart journal. 2007;154(5):995-1002.

15. Gerrior S, Juan W, Basiotis P. An easy approach to calculating estimated energy requirements. Preventing chronic disease. 2006;3(4):A129.

16. Davi G, Patrono C. Platelet activation and atherothrombosis. The New England journal of medicine. 2007;357(24):2482-94

17. Chu SG, Becker RC, Berger PB, Bhatt DL, Eikelboom JW, Konkle B, et al. Mean platelet volume as a predictor of cardiovascular risk: a systematic review and meta-analysis. Journal of thrombosis and haemostasis : JTH. 2010;8(1):148-56.

18. Kutlucan A, Bulur S, Kr S, Bulur S, Onder E, Aslantas Y, et al. The relationship between mean platelet volume with metabolic syndrome in obese individuals. Blood coagulation \& fibrinolysis : an international journal in haemostasis and thrombosis. 2012;23(5):388-90.

19. Tavil Y, Sen N, Yazici HU, Hizal F, Abaci A, Cengel A. Mean platelet volume in patients with metabolic syndrome and its relationship with coronary artery disease. Thrombosis research. 2007;120(2):245-50.

20. Toplak H, Wascher TC. Influence of weight reduction on platelet volume: different effects of a hypocaloric diet and a very low calorie diet. European journal of clinical investigation. 1994;24(11):778-80.

21. Coban E, Yilmaz A, Sari R. The effect of weight loss on the mean platelet volume in obese patients. Platelets. 2007;18(3):212-6.

22. Duffy BK, Gurm HS, Rajagopal V, Gupta R, Ellis SG, Bhatt DL. Usefulness of an elevated neutrophil to lymphocyte ratio in predicting long-term mortality after percutaneous coronary intervention. The American journal of cardiology. 2006;97(7):993-6.

23. Ryder E, Diez-Ewald M, Mosquera J, Fernandez E, Pedreanez A, Vargas R, et al. Association of obesity with leukocyte count in obese individuals without metabolic syndrome. Diabetes \& metabolic syndrome. 2014;8(4):197-204.

24. Agacayak E, Tunc SY, Sak S, Basaranoglu S, Yuksel H, Turgut A, et al. Levels of Neopterin and other Inflammatory Markers in Obese and Non-Obese Patients with Polycystic Ovary Syndrome. Medical science monitor : international medical journal of experimental and clinical research. 2015;21:2446-55.

25. Bahadir A, Baltaci D, Turker Y, Turker Y, Iliev D, Ozturk $\mathrm{S}$, et al. Is the neutrophil-to-lymphocyte ratio indicative of inflammatory state in patients with obesity and metabolic syndrome? Anatolian journal of cardiology. 2015;15(10):816-22. 